\title{
Intelligent Computing, Cognitive Graphics, Neural Networks in Computer Models of Forest Fires
}

\author{
Valery B. Taranchuk \\ Department of Computer Applications and Systems \\ Belarusian State University \\ Minsk, Republic of Belarus \\ taranchuk@bsu.by
}

\begin{abstract}
The results of creation of theoretical (mathematical), empirical (statistical), semi-empirical computer models of forest fires are discussed. The features of intelligent computing, obtaining accurate analytical (in the scope of empirical and semi-empirical models), approximate solutions (in theoretical models) are noted. For different models, examples of preprocessing of initial data, including the use of artificial neural networks, are noted. Presentable examples of illustrating the results of numerical experiments by tools of cognitive graphics using Wolfram Mathematica are given.
\end{abstract}

Keywords - Forest fire, computer model, intelligent computing.

\section{INTRODUCTION}

Of the many natural and anthropogenic factors affecting the state and dynamics of forest ecosystems, fires are dominant and cause significant material and environmental damage. Forest fires are divided into underground fires, surface fires, active crown fires, running crown fires, and mass fires. The strongest change in environmental condition parameters occurs in the zone called fire front, which propagates at some speed by the territory, covered with forest, and is visually observed as a zone captured by a flame. As a rule the effects of smoke generation over a big territory and clouds formation over a fire zone, because of condensation of the water vapour released during combustible forest materials burning, take place. A number of researchers notice, that running crown fires possess the greatest speed of propagation. They are most dangerous in the ecological means.

The development of appropriate computer models and software, their inclusion in the decision support systems for the prevention of emergency situations in forests and the surrounding area is required for reasonable, successful actions for the prevention, elimination of forest fires.

Below, as in a number of scientific publications [13], we will accept the following classification of computer models of forest fires: theoretical (mathematical), empirical (statistical), semi-empirical.

Interest in computer, mathematical models of forest fires, which are developed from the 1940s to the present time, is due to a large number of scientific and practical issues. There are some successes, but in many scientific publications it is noted that there are a number of problems.

In Belarus, much attention is paid to the problem of computer modelling of forest fires. In particular, the scientists of the Belarusian State University and the Institute of Mathematics of National Academy of Sciences of Belarus developed adapted theoretical and semi-empirical models of crown and surface forest fires. The program complexes of calculations of forecast characteristics for the conditions prevailing on the territory of the country forests are developed.

\section{THEORETICAL MODELS}

Theoretical models are based on the laws of continuum mechanics, other fundamental laws of physics and chemistry. Only they describe the formation of processes in dynamics taking into account general and local factors, inhomogeneities and allow answering a very wide range of questions. Mathematical descriptions of theoretical models are given, as a rule, in the form of a system of partial differential equations. Initial mathematical descriptions are usually written in dimensional variables, each law relates the values that determine the parameters of processes, the number of which is large, respectively. Usually the original system of mathematical descriptions has a large number of equations and expressions.

The development of theoretical models, the proposed numerical method for solving the corresponding boundary value problems for the modelling of crown and surface fires can be traced in articles $[4,5]$.

The creation of any computer model involves the analysis, the application of the theory of similarity and dimensions, reducing the number of equations, the correct formulation of the initial boundary value problem. The development of computer algebra systems (CAS) opens up new opportunities, and not only in the performance of various calculations, mathematical symbolic manipulations where you have to deal with conditions, complicated formulas, expressions. Now there is reason to say that artificial intelligence, CAS provides researchers with tools for automatically obtaining new formulations of initial boundary value problems. 
The development of theoretical computer models, performing with their use of computational experiments involves practical work with a computer in three different areas: pre-processing of the original data, the implementation of algorithms for calculating approximate solutions of the model equations, analysis of the results. Note the practical solutions that determine the effectiveness of the models $[2,4]$.

In part of preprocessing of the primary data used CAS Wolfram Mathematica significantly facilitates the starting analysis of the initial information. The corresponding tools of CAS are useful in the analysis of dimensions, the conversion of the values originally represented in different measurement systems, the transformation to dimensionless quantities $[6,7]$.

The classification and initial preparation of information for models involves extracting data from remote servers. For example, the above-mentioned models $[2,4,7]$ require characteristics of the forest and terrain, current weather conditions, including temperature, humidity, wind direction and speed, humidity for the previous 2 weeks. Technical aspects of such questions are considered and illustrated by examples in [7].

It is known that the data to be analyzed contain measurement errors, including system and random ones. In solving problems of mathematical modelling, the equations are written in differential form, so the original data (tooling of model) must be continuous, moreover, as a rule, the distribution should be smooth functions. Initially, the training used in theoretical models of distributions and functions were performed by specialists, and it took a lot of time. Currently, the software package includes and uses artificial neural networks. Examples of application of artificial neural networks, different variants of preprocessing and obtaining approximating smooth functions are presented in $[8,9]$.

The results of computer modelling, the examples of intelligent computing, the rules of formation of databases on the calculation protocols, research and forecasts of possible scenarios of crown forest fires propagation are presented in [2, 4, 5, 10-12].

It is also important to note that interactive visualization tools are used, and this significantly expands the possibilities of analyzing and interpreting the dynamics of processes. In particular, the analysis of the numerical results calculated on adapted model $[2,4]$ shows that two various scenarios of forest fire behaviour are possible: self-extinction and steady propagation. Using the computer model the question on boundaries, switch points of scenarios of fire process - steady propagation or extinction were studied (figure 1).

Whenever possible it is necessary to localize the switch line - one scenario on the one hand, another on the opposite. A study involves the calculation of variants in a wide range of determining parameters, the analysis of results defined in hyperspace (density of combustible forest materials, moisture content, equilibrium wind velocity) of sequence of points, fixing the scenario of the process.

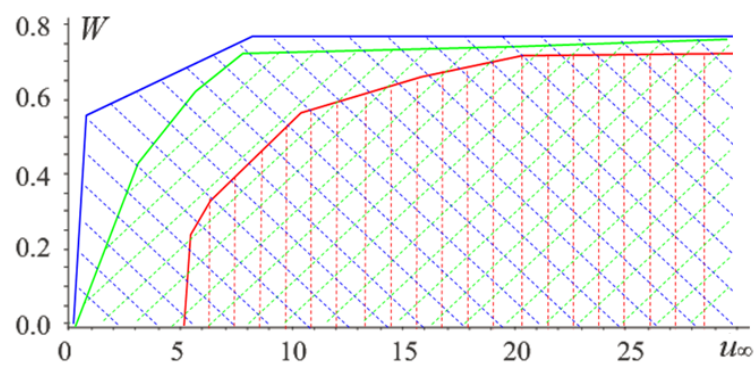

Fig. 1. Regions of steady fire propagation at several typical combustible forest materials layer density values. Results are received at $\rho 0: 0.1 \mathrm{~kg} / \mathrm{m} 3$ - shown as vertical lines, 0.2 - plus 45 inclinations, 0.4 - minus 45 inclinations.

A number of practical problems have been solved with the help of the developed software [10-12]. In particular, the dynamics of running crown fires in forests whose characteristics are not constant was modelled and analyzed. Corresponding conditions describe the practical challenges of forest fire fighting, such as the availability of forest buffer strips - strips free of forest vegetation (fuel breaks, rails, roads, watercourses), and fire barriers - strips with artificially increased water content, as well as strips of trees of non-combustible sorts. The executed numerical experiments allowed getting the width and length of a fuel break or fire barrier (and its moisture content) required for fire extinction.

The problems of computer modelling of surface forest fires under different conditions and for different configurations of forests were also considered [5], figures 2,3 . As in the case of running crown fires, it is principal to use interactive graphical visualization tools [13-15].

\section{SEMI-EMPIRICAL MODELS}

The empirical (statistical) models systematize data on the rate of spread of forest fire when changing the selected number of controlled parameters, determined by the correlation coefficients for each independent variable. Such an approach does not study the mechanism of the phenomenon; the resulting relations, strictly speaking, cannot be extended beyond the applicability of the statistics used, and within them a forecast is made with a certain probability. The issues of development and examples of application of such models can be found in [11].

In semi-empirical models, general laws (conservation of energy, mass and amount of motion) are used to determine the characteristics of fire propagation. They are written in the form of simplified dependencies, and the corresponding coefficients are selected by generalizing the experimental information. In comparison with empirical (statistical) semiempirical models are more adequate. Development and generalization of traditional models of this type is proposed in [11]. Semi-empirical models are adequate in situations similar to those in which experimental 
data were collected. Such models are much easier to verify than theoretical ones. Software realization of the proposed computer models of the above types, the technical aspects of their development and capabilities are described in [12].
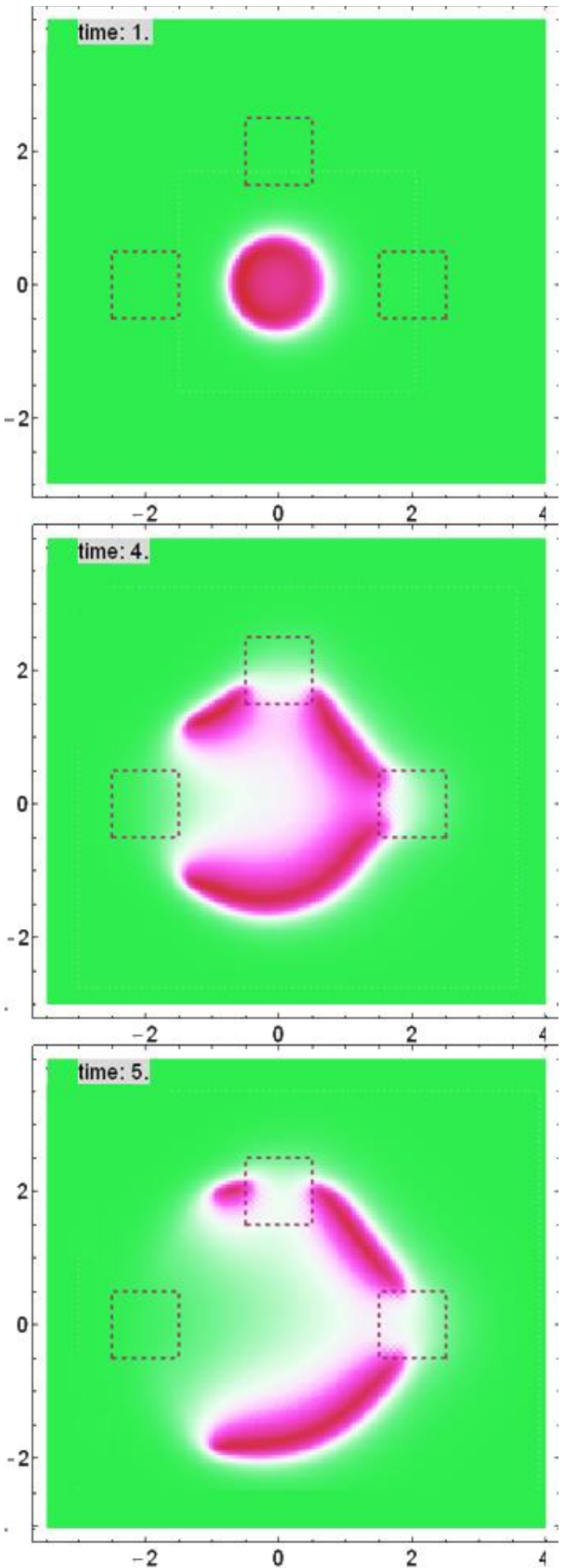

Fig. 2. The spread of fire in the presence of 3 glades, the wind from left to right, the speed of $1.5 \mathrm{~m} / \mathrm{s}$. The initial stage.
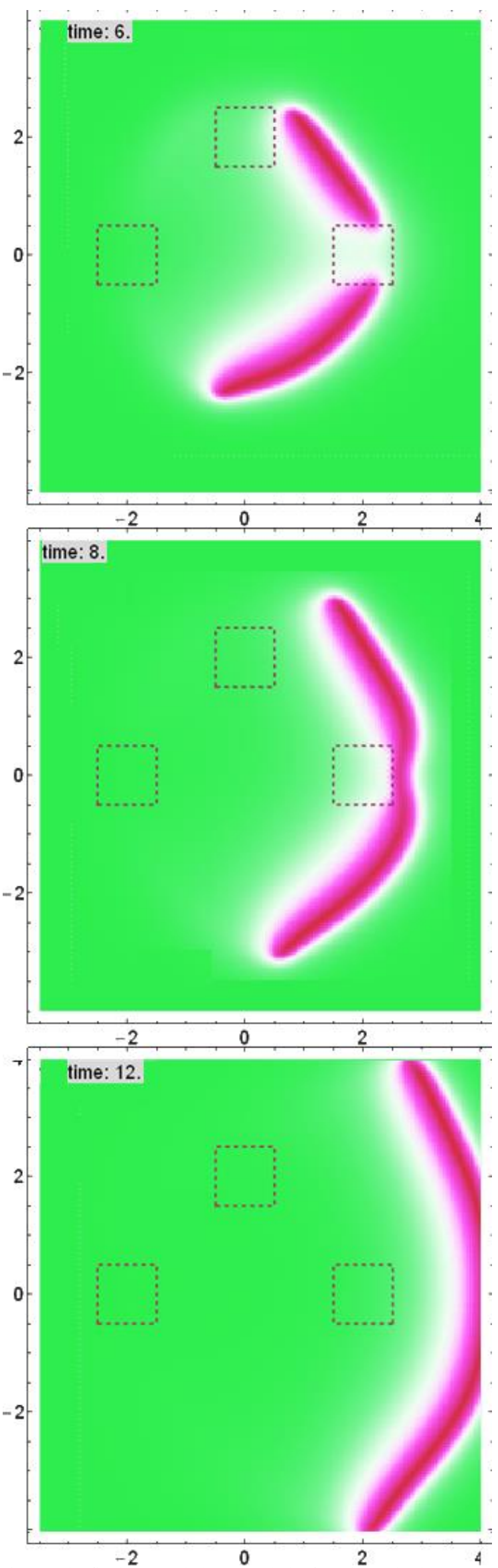

Fig. 3. The final stage, glades passed, the front is not broken. Upper and lower parts connected. 
Intelligent computing and cognitive graphics in semi-empirical models of forest fires. Consider examples of intelligent interactive computing and related graphical visualization. In this module, we note the options for the analysis and visualization of experimental data and results in research, preparation and justification of empirical and semi-empirical models for the case of working with the Rothermel's model [16]. The corresponding analysis was necessary and was carried out in the preparation and justification of generalization (modification) model [11]

The main mathematical component in the Rothermel's model is the proposed expression for determining the velocity of propagation of the fire front - equation (52). Consider the examples of execution in CAS Wolfram Mathematica analysis and comparison of several different possible options for the justification included in the equation (52) expression dimensionless parameter $\xi$, which connects the velocity of propagation of the front with the intensity of reaction. The corresponding dependence in $[16]$ is written in the form

$$
\begin{gathered}
\xi=(192+0.259 \sigma)^{-1} \\
\exp \left[\left(0.792+0.681 \sigma^{-0.5}\right)(\beta+0.1)\right]
\end{gathered}
$$

The basis of this expression is research and, in particular, the results of field experiments. Wolfram Mathematica allows you to interactively present and analyze similar results. Figure 4 left shows the illustration from [16] and to the right is a screenshot from Mathematica. Working with charts in CAS, you can select the work option in the drop-down menu [17]. You can build distribution maps on the plane $\sigma 0 \beta$ (2D graphics), you can display curves - family (1D graphics) $[18,19]$. In the 1D graphics, for example, we can use as argument $\sigma$ and study the family for different $\beta$. You can choose $\beta$ as an argument; see graphs of the family of curves by $\sigma$. In any of these options, you can use the slider on the scrolling panel to set any parameter value, in the user window the chart changes immediately $[20,21]$. In figure 4 , when you play back the results from [16] for a particular set, in which 3 data sets (shown on the left), you can view the specific values of the original laboratory data.

The user, leading the cursor to a specific point, sees the illumination of the corresponding data series, the values are highlighted to the left of the point. With tools for interactive visualization on the Rothermel's model you can make a few more refinements.

Basic. It should be noted that the expression $\xi(\sigma, \beta)$ is not only an interpolation or approximation of laboratory data. The corresponding analysis by means of cognitive graphics confirms the above, if we compare different variants of processing the experimental results. For example, figure 5 shows the dependence $\xi(\sigma, \beta)$ and the results of different interpolation options for the case $1 / 4$ " Cribs. When comparing Rothermel's curve with the curve obtained by interpolation of the third order, for clarity, the areas where the interpolating function below, filled in a light green, where the above yellow. Points with experimental data are shown, as in the original, by squares. Legend explains the approximation options: additionally, the results of interpolation of experimental data by moving average [22] and moving median [23] methods are given. The fragment in the lower right corner is detail graphics, namely, the insertion of the situation plan [24-26].
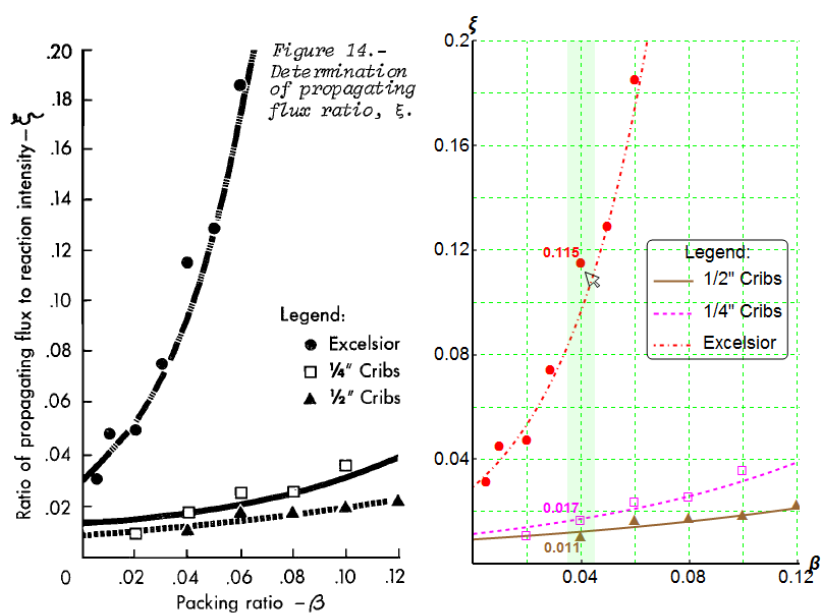

Fig. 4. Determination of propagating flux ratio, $\xi$.

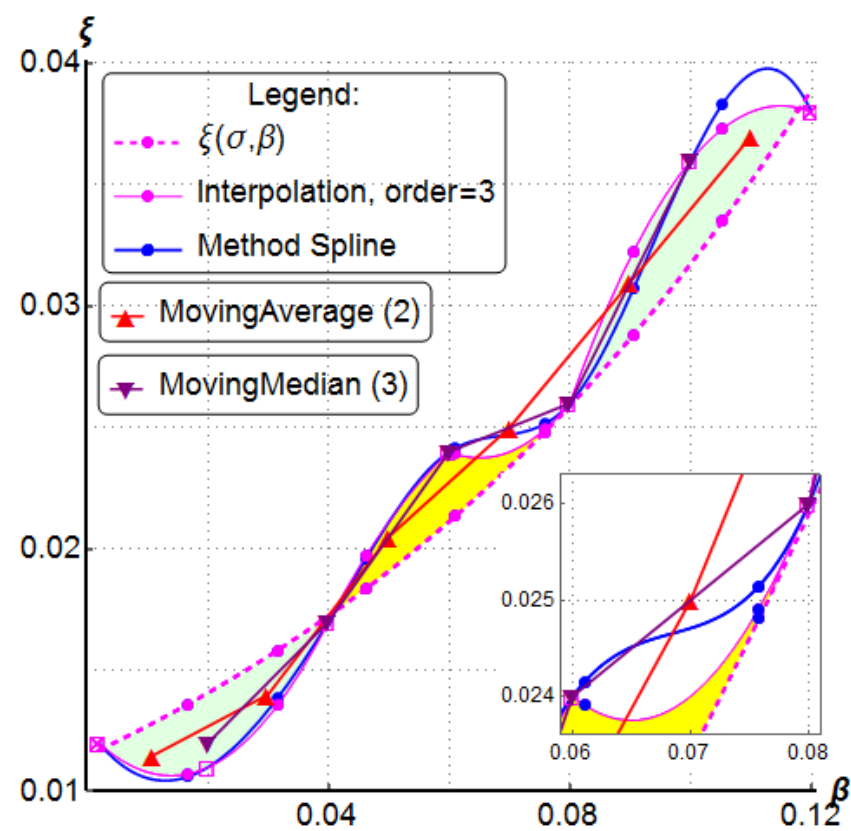

Fig. 5. The dependence $\xi(\sigma 2, \beta)$ and the results of different interpolation options. $\sigma 2$ corresponds to $1 / 4$ " Cribs.

\section{CONCLUSION}

The mentioned results of computer simulation of forest fires from the author's publications and the presented examples of interactive data processing demonstrate the high efficiency of the cognitive graphics system Wolfram Mathematica. 


\section{REFERENCES}

[1] Mathematical models and calculation systems for the study of wildland fire behaviour / E. Pastor [et al], in Progress in Energy and Combustion Science, 29, (2003), pp. 139-153.

[2] D.V. Barovik, V.B. Taranchuk, Sostoyanie problemy i rezul'taty komp'yuternogo prognozirovaniya rasprostraneniya lesnyh pojarov, in Vestnik BGU. Seriya 1, Fizika, Matematika, Informatika, 3 (2011), pp. 78-84 (in Russian)

[3] S.I. Marzaeva, O.V. Galtseva, A numerical solution of the problem of crown forest fire initiation and spread, in $I O P$ Conf. Series: Materials Science and Engineering, 363 (2018) 012020 doi:10.1088/1757-899X/363/1/0120202018

[4] D.V. Barovik, V.B. Taranchuk, Mathematical modelling of running crown forest fires, in Mathematical Modelling and Analysis, 15, 2, (2010), pp. 161-174.

[5] D.V. Barovik, V.I. Korzyuk, V.B. Taranchuk. K obosnovaniyu matematicheskih modelei nizovyh lesnyh pojarov, in Tr. In-ta matem., 21:1 (2013), pp. 3-14 (in Russian)

[6] CommonUnits - https://reference.wolfram.com/language/ref/ CommonUnits.html.

[7] Automatic Physical Units in Mathematica - https://blog wolfram.com/2010/12/09/automatic-physical-units-in-mathem atica.

[8] Valery Taranchuk, New computer technologies, analysis and interpretation of geodata, in MATEC Web of Conferences IPICSE-2018, 251, VI International Scientific Conference "Integration, Partnership and Innovation in Construction Science and Education" (IPICSE-2018), pp. 1-8.

[9] V.B. Taranchuk, Examples of the use of artificial neural networks in the analysis of geodata, in Open Semantic Technologies for Intelligent Systems, 3 (2019), pp. 225-230.

[10] D.V. Barovik, V.B. Taranchuk, Crown Forest Fire Mathematical Model Realization in Wolfram Mathematica, in Computer Algebra Systems in Teaching and Research. Mathematical Modeling in Physics, Civil Engineering, Economics and Finance, Poland, (2011), pp. 5-22.
[11] D.V. Barovik, V.B. Taranchuk, Adaptaciya modeli Rotermela dlya realizacii $\mathrm{v}$ programmnom komplekse prognoza rasprostraneniya lesnyh pojarov, in Nauchnyi internet jurnal Tehnologii tehnosfernoi bezopasnosti. 6, 40, (2011), pp. 1-8 (in Russian).

[12] D.V. Barovik, V.I. Korzyuk, V.B. Taranchuk, Metodicheskie i algoritmicheskie osnovy programmnogo kompleksa «Raschet i vizualizaciya dinamiki lesnogo pojara», in Chrezvychainye situacii: preduprejdenie i likvidaciya, 2, 30, (2011), pp. 22-33 (in Russian).

[13] ListContourPlot. - https://reference.wolfram.com/language/ ref/ ListContourPlot.html.

[14] ListDensityPlot. - https://reference.wolfram.com/language/ ref/ ListDensityPlot.html.

[15] Animate. - https://reference.wolfram.com/language/ref/Anim ate.html

[16] R.C. Rothermel, A Mathematical model for Predicting Fire Spread in Wildland Fuels in USDA Forest Service. Res. Pap. INT-115 (1972), 43 p

[17] PopupMenu. - https://reference.wolfram.com/language/ref/ PopupMenu.html

[18] Plot. - https://reference.wolfram.com/language/ref/Plot.html

[19] ListPlot. - https://reference.wolfram.com/language/ref/ListPl ot.html

[20] Manipulate. - https://reference.wolfram.com/language/ref/ Manpulate.html

[21] Slider. - https://reference.wolfram.com/language/ref/Slider. html.

[22] MovingAverage. - https://reference.wolfram.com/language/ ref/MovingAverage.html.

[23] MovingMedian. - https://reference.wolfram.com/language/ ref/ MovingMedian.html.

[24] PlotLegends. - https://reference.wolfram.com/language/ref/ PlotLegends.html.

[25] PointLegend. - https://reference.wolfram.com/language/ref/ Poi ntLegend.html.

[26] Epilog. - https://reference.wolfram.com/language/ref/Epilog. html 